\title{
UNA BREVE HISTORIA DE LA FILOSOFÍA COMO MODO DE VIDA Y LA ANTIFILOSOFÍA COMO SU HEREDERA CONTEMPORÁNEA
}

\author{
A Brief History of Philosophy as a Way of Life and Antiphilosophy \\ as its Contemporary Form
}

Alejandro Cavallazzi Sánchez, Universidad Iberoamericana, México

Correo electrónico: alejocava@gmail.com

Recepción: 10/04/2019

Aceptación: 21/01/2020

Resumen. Se presenta la concepción de la filosofía como modo de vida según la obra de Pierre Hadot, desde su comprensión en la Antigüedad hasta su incorporación a la religión cristiana en la Edad Media. La antifilosofía, propuesta de Jacques Lacan y, posteriormente, desarrollada por Alain Badiou, constituye un discurso distinto a la filosofía que bien puede entenderse como el modo de vida desarrollado por Hadot. La antifilosofía puede comprenderse como el discurso heredero de la propuesta de Hadot: una forma de entender el pensamiento como un modo de vida en tiempos contemporáneos. En este trabajo mostraremos un breve recuento de la evolución de la filosofía como modo de vida y cómo la antifilosofía retoma estas inquietudes en nuestro tiempo.

Palabras clave: antifilosofía, Hadot, Lacan, Badiou, historia.

\begin{abstract}
The conception of philosophy is presented as a way of life according to Pierre Hadot's work. From his understanding in antiquity to its incorporation into Christianity in the Middle Ages. The antiphilosophy, proposed by Jacques Lacan and subsequently developed by Alain Badiou, constitutes a discourse different from the discourse of philosophy. Antiphilosophy can well be understood as the "way of life" developed by Hadot. Antiphilosophy can be understood as the heir discourse of Hadot's proposal: a way of understanding thought as a way of life in contemporary times. In this work we will show a brief account of the evolution of philosophy as a way of life and how antiphilosophy takes up these concerns in our time.

Keywords: Antiphilosophy, Hadot, Lacan, Badiou, History
\end{abstract}


La filosofía antigua propone al hombre un arte de vivir, al contrario que la moderna, que aboga en primer lugar por la construcción de un lenguaje técnico reservado a especialistas.

Pierre Hadot, Ejercicios espirituales y filosofia antigua

\section{¿Qué es la filosofía como modo de vida en la filosofía clásica?}

Pierre Hadot dedicó buena parte de su obra a mostrar que la filosofía en la Antigüedad debe entenderse no sólo como un discurso teórico sino también como un modo de vida ${ }^{1} \mathrm{y}$ que, incluso, este modo de vida representaba lo más importante para los filósofos antiguos. $^{2}$ "La Antigüedad entiende la filosofía como un ejercicio a practicar constantemente; invita a concentrarse en cada instante de la existencia, a tomar consciencia del infinito valor del momento presente siempre que uno lo sitúe en una perspectiva cósmica". ${ }^{3}$ Esta perspectiva cósmica implica que el filósofo antiguo buscaba situarse a sí mismo y a sus contemporáneos en una relación concreta y efectiva con la naturaleza en vista de un mejoramiento personal. Hadot argumenta que

podemos lograr una conciencia cósmica que nos eleva por encima de las pequeñas preocupaciones de nuestras vidas individualistas y nos hace conscientes de que somos partes del todo. Este objetivo final es equivalente a la

\footnotetext{
1 “La afirmación más citada de Hadot es que la filosofía antigua era una forma de vida, y basa esta opinión en una reconstrucción cuidadosa y objetiva del contexto de los textos fílosóficos antiguos" ["Hadot's most often quoted claim is that ancient philosophy was a life choice, and hist support for this claim is based on careful, objective reconstruction of the context of ancient philosophical texts"]. Pierre Force, "The Teeth of Time: Pierre Hadot on Meaning and Misunderstanding in the History of Ideas", History and Theory 50, núm. 1 (febrero, 2011): 20-40.

2 "El filósofo francés Pierre Hadot identificó un componente faltante en el mundo de la filosofía moderna. $\mathrm{Su}$ gran innovación se produjo en la identificación de la filosofía como un modo de vida, acompañado de ciertos ejercicios espirituales, una visión derivada de su estudio de la filosofía antigua. En este entendimiento, sólo cuando vivían su filosofía, los filósofos podían llamarse filósofos. Hadot cita repetidamente a Henry David Thoreau: 'Hoy en día, hay profesores de filosofía, pero no filósofos" [The French philosopher Pierre Hadot identified a component missing from the world of modern philosophy. "His great innovation came in identifying philosophy as a "way of life," accompanied by certain "spiritual exercises," an insight derived from his study of ancient philosophy. In this understanding, only when they lived their philosophy could philosophers properly be called philosophers. Hadot repeatedly quotes Henry David Thoreau: "Nowadays, there are philosophy professors, but no philosophers"]. Matthew Kruger, "Aquinas, Hadot, and Spiritual Exercises", New Blackfriars 98, núm. 1075 (2017): 414.

${ }^{3}$ Pierre Hadot, Ejercicios espirituales y filosofía antigua. Biblioteca de Ensayo (Madrid: Siruela, 2006), 246-247.
} 


\section{enn-claves}

felicidad, en el sentido dado a este concepto en la filosofía helenística: liberarse de la ansiedad, la angustia, las preocupaciones y la desesperación. ${ }^{4}$

La búsqueda de la filosofía antigua se centraba en la transformación del individuo. Entonces la filosofía buscaba una conversión conducente que abonara no en el pensamiento sino en el vivir. Para Hadot la filosofía, en su sentido más profundo y esencial era "una actividad de elección y de decisión cuya responsabilidad asume sólo el individuo". 5

Para Hadot la filosofía se comprende en dos modalidades. Por un lado, el discurso filosófico que Hadot entiende como la forma en que se divulgaban las diferentes doctrinas y se hacían explícitos los supuestos teóricos de cada postura. La segunda como modo de vida que constituían ante todo el arte de vivir, esto es un compromiso vital, una toma de postura a partir de las reflexiones generadas en el discurso filosófico. El contenido teórico era "incapaz de expresar lo que es esencial: para Platón, las formas, el bien, es decir, lo que se experimenta, de una manera no discursiva, en el deseo y en el diálogo". ${ }^{6}$ Como se observa en diversos pasajes de la obra platónica, paradigmáticamente en El Banquete, el discurso apunta a la acción. El traslado de la teoría a la acción se realizaba ayudado de ciertas prácticas que Hadot bautiza con el nombre de ejercicios espirituales. Una de ellas consistía en reflexionar sobre la finitud de la vida. Por algo, para Platón, el ejercicio de pensar la muerte resumía la esencia misma de la filosofía. ${ }^{7} \mathrm{La}$ filosofía debe conducir a una forma de vivir, una vida recta y una preparación para la muerte. “'Filosofar es aprender a morir' supone una de las frases más adecuadas que se hayan acuñado nunca para definir la filosofía". ${ }^{8}$ Los ejercicios espirituales se practicaban bajo el contexto de la doctrina. Todo el quehacer filosófico debe conducir a la acción.

\footnotetext{
4 "By all these means, Hadot argues, we can achieve a cosmic consciousness that raises us above the petty concerns of our individualistic lives, and makes us aware that we are parts of the All. This final goal is equivalent to happiness, in the sense given to this concept in Hellenistic philosophy: freedom from anxiety, anguish, worries, and despair". Michael Chase, "Observations on Pierre Hadot's Conception of Philosophy as a Way of Life", Philosophy as a Way of Life: Ancients and Moderns - Essays in Honor of Pierre Hadot. Editado por Michael Chase, Stephen R. L. Clark, Michael McGhee (Hoboken, NJ: Wiley-Blackwell, 2013), 264.

${ }^{5}$ Pierre Hadot, ¿Qué es la filosofia antigua? (México: Fondo de Cultura Económica, 1998), 158-159.

${ }^{6}$ Ibid., 89.

${ }^{7}$ Cfr. Ibid., 227.

${ }^{8}$ Hadot, Ejercicios espirituales, p. 302.
} 


\section{enn-claves}

Pero la filosofía en sí misma, es decir, la forma de vida filosófica, no consiste tanto en una teoría dividida en partes como en una actividad exclusiva dirigida a vivir la lógica, la física y la ética. No se teoriza entonces sobre lógica, es decir, sobre hablar y pensar correctamente, sino que se piensa y se habla bien, no se teoriza sobre el mundo físico, sino que se contempla el cosmos, ni tampoco se teoriza sobre la acción moral, sino que se actúa de manera recta y justa. ${ }^{9}$

Es a través de un cambio de perspectiva, de una transformación de nuestra forma de ver el mundo que la filosofía permite la transformación individual. ${ }^{10}$ Según Hadot el filósofo de la Antigüedad era aquel que podía llevar su modo de vida a la altura de los discursos lo cual dista de la comprensión contemporánea del sabio como un erudito coleccionador de datos. En nuestro tiempo la formación filosófica se entiende primordialmente como la lectura y la interpretación de textos filosóficos. En la Antigüedad, en cambio, la textualidad era un recurso adicional para la, entonces, considerada verdadera forma filosófica: la expresión oral. Si pensamos que los diálogos de Platón estaban destinados a ser presentados en lecturas públicas. ${ }^{11}$ En la Antigüedad "la verdadera formación es siempre oral, ya que sólo la palabra permite el diálogo, es decir la posibilidad para el discípulo de descubrir él mismo la verdad en el juego de preguntas y respuestas, la posibilidad también para el maestro de adaptar su enseñanza a las necesidades del discípulo". ${ }^{12}$

Para los antiguos, el discurso filosófico hablado es muy superior al discurso filosófico escrito. ${ }^{13}$ "A los ojos de Platón, el ejercicio del diálogo era más importante que los resultados obtenidos en dicho ejercicio. Así mismo, para Aristóteles, la discusión de los problemas es finalmente más formada que la solución". ${ }^{14}$ Entonces, el diálogo era personalizado y se dirigía a un público muy específico pensando en sus necesidades. Hadot nos amplía: "esto me condujo a la idea de que las obras filosóficas de la Antigüedad no se componían para exponer un sistema, sino para producir un efecto de

\footnotetext{
${ }^{9}$ Ibid., 238.

10 "By changing our way of looking at the world, we are to transforming ourselves to the point of becoming fully integrated beings, mastering our internal discourse in the way a rhetorician masters external discourse in his speeches, harmonizing our will and desires with the course of Nature, and recognizing and fulfilling the social obligations placed upon us by the demand for Justice". Chase, "Observations", 264.

${ }^{11}$ Cfr. Pierre Hadot, La filosofía como forma de vida, conversaciones con Jeannie Carlier y Arnold I. Davidson (Barcelona, Alpha Decay, 2009), 89.

${ }^{12}$ Pierre Hadot, Elogio de la filosofía antigua (México: Me Cayó el Veinte, 2009), 56.

${ }^{13}$ Cfr. Pierre Hadot, ¿Qué es la filosofía antigua? (México Fondo de Cultura Económica, 1998), 84.

${ }^{14}$ Ibid., pp. 101-102.
} 


\section{enn-claves}

formación: el filósofo quería hacer trabajar los espíritus de sus lectores o auditores para ponerlos en una disposición determinada". ${ }^{15}$ Ya fuera a través de su estilo de vida o por medio del discurso "la idea más importante de Hadot sobre los filósofos antiguos aparece así: que su objetivo era, en palabras de Victor Goldschmidt; formar, más que informar". ${ }^{16}$

Cuando se enseñaba la doctrina filosófica en la Antigüedad no se trataba de presentar puntos específicos de sistemas de pensamiento o interpretaciones intelectuales sino de formar a los participantes y promover una nueva forma de vivir. No se buscaba resolver problemas teóricos y abstractos sino "formular, siempre de nuevo y de manera llamativa, máximas que inviten a la acción; lo que cuenta es el acto de escribir, de hablarse a uno mismo". ${ }^{17}$

De tal magnitud era la importancia que se le concedía al modo de vivir que los filósofos de la Antigüedad sospechaban de quienes emitían elaborados discursos retóricos sin sustentarlos con una comprometida forma de vivir pues "no se trata solamente de vanidad sofística, de pequeña vanagloria, del placer hablar". ${ }^{18}$ Así, existían concepciones opuestas de la filosofía,

una que ponía el acento en el polo del discurso, y otra que lo ponía en el polo de la elección de vida. Ya en la Antigüedad, sofistas y filósofos se enfrentaban. Los primeros intentaban brillar por las sutilezas de la dialéctica o la magia de las palabras, los segundos pedían a sus discípulos un compromiso concreto con una determinada forma de vida. ${ }^{19}$

Según Hadot el componente que distinguía entonces a la filosofía de la sofística no consistía en adoptar una cierta postura intelectual sino precisamente en adquirir el

\footnotetext{
${ }^{15}$ Hadot, La filosofia, 99.

16 "Hadot's most important insight about ancient philosophers comes in: that their goal was, in Victor Goldschmidt's words, "to form, more than to inform"”. en Force, "The Teeth of Time", 27. Al respecto confrontar con lo que dice el propio Hadot: "V. Goldschmidt, de quien no se podría sospechar que deseara minimizar el aspecto sistemático de las doctrinas, propuso la mejor explicación de este hecho al decir que los diálogos no fueron escritos para 'informar', sino para 'formar'. Ésta es, asimismo, la intención profunda de la filosofía de Platón, que no consiste en construir un sistema teórico de la realidad y en 'informar' luego a sus lectores, escribiendo una serie de diálogos que expongan en forma metódica este sistema, sino que estriba en 'formar', es decir, en transformar a los individuos, haciéndoles experimentar en el ejemplo del diálogo al que el lector tiene la ilusión de asistir, las exigencias de la razón y finalmente la norma del bien". Hadot, ¿Qué es la filosofía antigua?, 86.

${ }^{17}$ Ibid., 195.

${ }^{18}$ Hadot, La filosofia, 175.

${ }^{19}$ Ibid., 100.
} 


\section{en-claves}

compromiso de vivir filosóficamente. Para el sofista esto era trivial y no tenía cabida en su discurso mientras que para el filósofo era justamente lo esencial.

\section{Primer cambio de paradigma: enseñanza de la filosofía en el periodo romano}

El propósito de los textos filosóficos y el modo en que se conducía su lectura en la Antigüedad dista mucho del modo en que se hace actualmente. Se discutía alguna pregunta de carácter filosófico como ¿qué es el bien?, ¿cuál es el placer supremo? o ¿cuál es una vida digna de ser vivida?, pero con la intención de llevar estas dudas a la acción.

Esta forma de discurso [la discusión de las tesis] era habitual entre los antiguos, y de esta manera impartían sus clases, no comentando libros como sucede ahora (en efecto, en esa época, no había libros de ese tipo), sino, habiendo sido planteada una tesis, argumentaban en favor o en contra, para ejercitar su facultad de inventar argumentaciones, apoyándose en premisas admitidas por todo el mundo. ${ }^{20}$

Los textos antiguos eran herramientas de estudio para conducir las lecciones a través del diálogo y la discusión, no para ser leídos en solitario o ser analizados exegéticamente al modo contemporáneo. La finalidad de las doctrinas de los filósofos era "reunir en forma condensada los dogmas fundamentales, y entrelazarlos por medio de una argumentación rigurosa, a fin de crear un núcleo sistemático muy concentrado, a veces hasta condensado en una breve sentencia, que tendrá así una mayor fuerza persuasiva, una mejor eficacia mnemotécnica". ${ }^{21} \mathrm{Si}$ existía entonces una sorprendente continuidad en los principios intelectuales y dogmas entre las escuelas de la Antigüedad se debe a que éstos servían como guías para lo considerado realmente importante que era elegir un modo de vida. El discurso teórico de la filosofía antigua no era sino un recurso para acceder a la verdadera esencia de la disciplina. Hadot refiere que ésta era la misión de toda la filosofía: las escuelas más populares como el epicureismo o estoicismo se dirigían a todos los hombres, ricos o pobres, hombres o mujeres, libres o esclavos, mientras que otras escuelas más sofisticadas, como el platonismo o el aristotelismo, eran reservadas a

\footnotetext{
${ }^{20}$ Alejandro de Afrodisias. Comentarios a los Tópicos de Aristóteles, citado en Hadot, ¿Qué es la filosofía antigua?, 119.

${ }^{21}$ Hadot, ¿Qué es la filosofia antigua?, 121. 


\section{en-claves}

Revista de Filosofía, Arte, Literatura, Historia

e-ISSN: 2594-1100, Año XIV, Núm 27, enero-junio 2020, 1-18

una élite que disponía de tiempo libre. ${ }^{22}$ No obstante, estas diferencias la labor de la filosofía era la misma para todas las escuelas: conducir a los individuos a mejorar su propia existencia.

Esta misión se mantuvo por siglos. La enseñanza filosófica mantuvo su preferencia por la pedagogía oral gracias a la continuidad de las instituciones académicas como la Academia o el Liceo hasta el año 87 a.C. y la destrucción de Atenas a manos del general romano Lucio Cornelo Sila. Las escuelas filosóficas reaparecen en Alejandría y Roma, pero en estos lugares se pierde el sentido clásico de hacer filosofía y con ello el sentido en que se leían los textos.

En lo sucesivo, la enseñanza consistirá en explicar los textos de las 'autoridades', por ejemplo, los diálogos de Platón, los tratados de Aristóteles, las obras de Crisipo y de sus sucesores. En la época anterior, la actividad escolar consistía ante todo en habituar a los alumnos a métodos de pensamiento y de argumentación. ${ }^{23}$

Atrás queda la discusión clásica de las tesis con un fin edificante, la lectura teatral de los diálogos y la enseñanza de la filosofía como un modo de vida. En esta época romana la filosofía se convierte en algo que nosotros reconoceríamos con facilidad: una disciplina más bien académica y escolar; la enseñanza misma de la filosofía adquiere la forma de un comentario a un texto.

En la época romana prosperan los manuales, los comentarios y los resúmenes que busca exponer la teoría de los grandes filósofos. "Los aprendices de filósofo a menudo se inclinaban a interesarse más en el perfeccionamiento de su cultura general que en la elección de vida existencial que supone la filosofia". ${ }^{24} \mathrm{Si}$ bien todavía permanece algo del carácter espiritual de la filosofía griega, como lo atestiguan las doctrinas de los estoicos, sin embargo, la enseñanza de la filosofía será transformada de forma definitiva. Lo que fuera alguna vez la pedagogía filosófica como discusiones que hoy llamaríamos vitalistas o existenciales de los antiguos griegos en ese entonces se convertirían en pura exégesis.

\footnotetext{
${ }^{22}$ Cfr. Ibid., 122.

${ }^{23}$ Ibid., 165.

${ }^{24}$ Ibid., 166.
} 


\section{Segundo cambio de paradigma: el cristianismo y la aparición de la universidad medieval}

La aparición del cristianismo representó una segunda transformación de la pedagogía y los alcances de la filosofía. Los propios cristianos presentaron su doctrina como una filosofía única, verdadera y eterna. Los apologistas recurrieron al concepto filosófico de Logos para definir el cristianismo como filosofía. Hadot cita a Justino: "Si filosofar supone vivir en conformidad con la ley de la Razón el cristiano es un filósofo, puesto que vive en conformidad con la ley del Logos, de la Razón divina". ${ }^{25}$ Este Logos cristiano que suele traducirse como verbo en la tradición religiosa y razón en la filosófica no debe comprenderse sólo como una o la otra sino ambas. El Logos es en efecto razón y doctrina, pero no sólo eso sino, como sucedía en la filosofía clásica, también se trata de un modo de vida.

Si los cristianos pudieron tomar la palabra philosophia para designar esa perfección de la vida cristiana que representa el monaquismo, se debe a que la palabra philosophia significaba en efecto un modo de vida, de tal manera que al conservar la palabra los 'filósofos cristianos' se vieron llevados a introducir en el cristianismo prácticas y actitudes heredadas de la filosofía profana. No tiene por qué sorprender: la vida filosófica profana y la vida monástica tenían en el fondo muchas analogías. ${ }^{26}$

Se da aquí una convergencia de objetivo e incluso una duplicación de funciones. Los primeros monjes imitaron las prácticas filosóficas de los ejercicios espirituales. Las prácticas estoicas sobre el dominio de sí coinciden con la ascética monástica que buscaba el sometimiento de las pasiones. Por esta razón los cristianos primitivos incorporan estas prácticas, pero otorgándoles un talante religioso. "Según Evagro, la muerte en la que se adiestra ese filósofo que es el monje es, pues, la total extirpación de las pasiones que

\footnotetext{
${ }^{25}$ Justino, Apología, citado en Hadot, Ejercicios espirituales, 241.

${ }^{26}$ Hadot, ¿Qué es la filosofia antigua?, 268.
} 


\section{enn-claves}

unen el alma al cuerpo, a fin de alcanzar el perfecto desprendimiento del cuerpo que proporciona la apatheia, la ausencia de pasiones". ${ }^{27}$

El cristianismo logró realizar lo que sólo era una lejana aspiración de las escuelas filosóficas: el llamado a la población general a una conversión y una mejor forma de vida. Para Agustín esto constituye la diferencia entre cristianismo y platonismo, y da cuenta de la superioridad de la religión. "El platonismo no pudo convertir a las masas y alejarlas de las cosas terrestres para orientarlas hacia las espirituales, en tanto que, desde la llegada de Cristo, hombres de toda condición adoptaron este modo de vida y presenciamos una verdadera transformación de la humanidad". ${ }^{28}$

El monaquismo incorporó los ejercicios espirituales filosóficos como parte de su modo de vida, esto aunado a los ritos propiamente cristianos, y con la extinción de las escuelas de filosofía se convirtió en el sitio exclusivo para estas prácticas. Las instituciones académicas desaparecieron y las doctrinas fueron aniquiladas o incorporadas al dogma. La filosofía dejó de ser transmitida como un modo de vida en las diferentes escuelas y sobrevivió de manera independiente como un repositorio de contenidos intelectuales separada de los modos de vida que alguna vez le inspiraron.

Con el cristianismo se separan dos configuraciones que habían constituido hasta entonces una unidad inseparable en la filosofía: discurso y modo de vida. A partir de entonces la religión se apropia tanto de los ejercicios espirituales como del carácter edificante de la filosofía clásica que apelaba a la conversión personal e interior. La filosofía conserva lo único que le queda: el discurso y se dedica a los objetivos exclusivamente teóricos a los que estamos acostumbrados hoy en día. En otras palabras, la aparición y el éxito del cristianismo supone la reducción de la filosofía de un modo de vida a un discurso teórico.

En las universidades de la Edad Media, se puso fin a la confusión existente primitivamente en el cristianismo entre la teología, fundamentada en la fe, y la filosofía tradicional, fundamentada en la razón. La filosofía deja de considerarse la ciencia suprema, pasando a ser 'sierva de la teología'; ella le suministra los materiales conceptuales, lógicos, físicos o metafísicos de que

\footnotetext{
${ }^{27}$ Ibid., 267.

${ }^{28}$ Ibid., 272.
} 


\section{enn-claves}

ésta tiene necesidad. La facultad de artes se entiende como una simple preparación para la facultad de teología. ${ }^{29}$

A partir de entonces la enseñanza de la filosofía ya no tiene como vocación formar hombres sino especialistas. Ésta es la característica en común que tienen todas las corrientes escolásticas. La filosofía se considera entonces no más que un discurso teórico y al ser disociada de su comprensión esencial como modo de vida pasa a ser considerada sierva de la teología. Esta característica, que no era lo esencial de la filosofía, permanecerá desde entonces con la disciplina. Para cuando "la filosofía moderna conquiste su autonomía, en el siglo XVII, y sobre todo en el XVIII, siempre tendrá tendencia a limitarse a este punto de vista". ${ }^{30}$ A partir de entonces, la filosofía deja de entenderse como una forma de vida, "a menos que por eso se entienda el género de vida del profesor de filosofía". 31

A partir de la aparición de la teología la filosofía debe conformarse con ser "una ciencia teórica, porque la dimensión existencial de la filosofía ya no tenía sentido desde la perspectiva del cristianismo, que era al mismo tiempo doctrina y vida". ${ }^{32}$ Desde entonces la filosofía es incorporada a la universidad; supeditada a las necesidades educativas de los tiempos que, en ocasiones, siguen una agenda política o económica nacional. Por este motivo las instituciones terminan por hacer

del profesor de filosofía un funcionario cuyo oficio consiste, en gran parte, en formar a otros funcionarios; ya no se trata, como en la Antigüedad, de formar para el oficio de hombre, sino para el de clérigo o de profesor, es decir de especialista, de teórico, poseedor de cierto conocimiento, más o menos esotérico. Pero este saber ya no pone en juego toda la vida, como lo deseaba la filosofía antigua. ${ }^{33}$

La crítica de Hadot resuena con ciertos pensadores del siglo XIX que se encontraban al margen de la academia como lo fueron Kierkegaard, Nietzsche o Schopenhauer. Si atendemos a sus obras encontraremos una concepción similar a la de Hadot donde el "filosofar o pensar es y debe ser un asunto existencialmente serio, ligado

\footnotetext{
${ }^{29}$ Hadot, Ejercicios espirituales, 242.

${ }^{30}$ Hadot, ¿Qué es la filosofía antigua?, 274.

${ }^{31}$ Hadot, Ejercicios espirituales, 244.

${ }^{32}$ Hadot, ¿Qué es la filosofía antigua?, 280.

${ }^{33}$ Ibid., 282.
} 


\section{enn-claves}

íntimamente a los intentos de los individuos de vivir bien". ${ }^{34}$ Kierkegaard, por ejemplo, fue crítico con las instituciones educativas y la figura pública del profesor universitario: "de todos los repugnantes inhumanos, el más repugnante". ${ }^{35}$ Así estos "pedantes doctorales. Viven en esta vida, instalados en sus pensamientos; gozan de una situación sólida y de opiniones seguras en un Estado bien organizado; han puesto por medio siglos, por no. decir milenios, entre ellos y los tremendos avatares de la existencia". ${ }^{36}$ Para Kierkegaard, los profesores universitarios se dan a la tarea de juzgar a los grandes hombres sin que ellos siquiera se hayan preguntado alguna vez sobre su propia existencia. ${ }^{37}$ Qué distinto resulta esta posición del filósofo antiguo que se jugaba su posición de maestro a cada momento de acuerdo a la manera en que conducía su propia vida.

\section{La antifilosofía como herencia de la filosofía como modo de vida}

¿Queda algo del espíritu de la filosofía como un modo de vida en la filosofía de nuestro tiempo? Queremos argumentar que, si bien parece muy difícil que la figura del filósofo contemporáneo comparta las características del sabio filósofo de la Antigüedad, un estilo de pensamiento conocido como antifilosofía ${ }^{38}$ rescata los intereses de la filosofía como un modo de vida.

Jacques Lacan definió a la filosofía como aquella área de estudio de los que se ha expuesto al psicoanálisis en el contexto de una educación universitaria $;{ }^{39}$ un discurso que pretende ser educativo pero que termina siendo, al fin y al cabo: institucional. ${ }^{40}$ Para

\footnotetext{
34 "For both, philosophising or thinking is and ought to be an existentially serious affair, tied intimately with individuals' attempts to live well". Matthew Sharpe, "Socratic Ironies: Reading Hadot, Reading Kierkegaard", Sophia 55 (2016): 431.

${ }^{35}$ Soren Kierkegaard, Journals and Papers (Bloomington, IN: Indiana University Press, 1978), XI, A 412, 1854 .

${ }^{36}$ Soren Kierkegaard, Temor y temblor (Madrid: Alianza Editorial, 2001), 121.

${ }^{37}$ Cfr. "La misión de juzgar a los grandes hombres de acuerdo con los resultados que hayan obtenido. Semejante comportamiento frente a lo grandioso delata una extraña mezcla de soberbia y miseria; soberbia por considerarse llamados a juzgar, y miseria porque no sienten en los más mínimo emparentadas sus existencias con las de los grandes hombres", Kierkegaard, Temor y temblor, 121.

${ }^{38}$ Para un tratamiento ampliado de este tema véase Alejandro Cavallazzi, "¿Qué es Antifilosofía?", LÍMITE Revista Interdisciplinaria De Filosofía Y Psicología 14, núm. 2 (2018), https://www.revistalimite.cl/index.php/limite/article/view/108.

${ }^{39}$ Adrian Johnston, "This Philosophy Which Is Not One: Jean-Claude Milner, Alain Badiou, and Lacanian Antiphilosophy", Journal of the Jan van Eyck Circle for Lacanian Ideology Critique 3 (2010): 137.

${ }^{40}$ Jacques Lacan, "Quizás en Vincennes”, en Otros escritos (Buenos Aires: Paidós, 2012), 335.
} 


\section{enn-claves}

Lacan, la filosofía sería una forma de conocimiento que se mantiene sujeto de la necesidad o del deseo de un poder político. ${ }^{41}$ El filósofo profesional dice lo que el amo calla, opera como el bufón de la corte ante el rey. El filósofo gira y danza alrededor del discurso del maestro. Él juega al papel del loco pues dice lo que nadie más puede, pero siempre con la intención de servir al rey. ${ }^{42}$ Observamos esta dinámica en la figura del profesor universitario que transmite sin pasión alguna su conocimiento y se vuelve un recurso más de la institución universitaria. En la opinión de Jean-Claude Milner, uno de los más célebres intérpretes de Lacan, el profesor se vuelve algo perfectamente sustituible ya que lo que le autoriza como docente no tiene nada que ver con su compromiso como educador sino con una posición académico-burocrática. ${ }^{43} \mathrm{El}$ profesor universitario sólo es dueño de ocupar impersonalmente el lugar del saber. Su diferencia con el maestro de los antiguos es que su docencia no está en juego con su forma de vida. ${ }^{44}$

Ante la perspectiva de una filosofía burocrática y excluyente, Lacan opone su propio discurso con fines terapéuticos y le bautiza con el término antifilosofía. Esta antifilosofía lacaniana no debe considerarse como un asalto contra la disciplina filosófica sino contra la profesionalización del discurso académico. ${ }^{45}$

Cabría la pregunta de si la antifilosofía de Lacan puede equiparse con la comprensión de la filosofía como un modo de vida según Hadot. A este respecto se puede consultar el trabajo de Gwenaëlle Aubry, una de las discípulas de Hadot, quien tuvo la oportunidad de asistir a los seminarios sobre antifilosofía de Badiou mientras examinaba su manuscrito de ¿Qué es la filosofía antigua? A partir de esa coincidencia se hizo la pregunta sobre la similitud entre antifilosofía y modo de vida, concluyendo que se tratan

\footnotetext{
41 "Lacan's third claim concerning philosophy in The Other Side of Psychoanalysis is thus to associate philosophy with what he from this time now delineates as 'the discourse of the masters'. Philosophy would on this account be a way of seeking knowledge which, despite Plato's famous claim that the philosopher should ascend out of the political-historical cave (Rep. VII), would always remain subject to the needs of, if not the desire for, political rule (in the matheme of the discourse of the master, the S1 in the position of agency)". Matthew Sharpe, "Killing the father, Parmenides: On Lacan's Anti-philosophy", Continental Philosophy Review 52 (2019): 51-74.

42 "Le philosophe est donc celui qui tourne rond dans le discours du maître. Il y joue le rôle du fou. C'est-àdire le tenant-lieu de la vérité, absolument inconscient de ce qu'il dit, et qui, par conséquent, n'est astreint qu'à tenir ce rôle". Alain Badiou, Le Seminaire. Lacan. L'antiphilosophie 3. 1994-1995 (París: Librairie Arthème Fayard, 2013), 22.

${ }^{43}$ Adam Rodolphe, Lacan y Kierkegaard (Buenos Aires: Nueva Visión, 2007), 285.

${ }^{44}$ Ibid., 285.

${ }^{45}$ Cfr. Jacques Lacan, El Seminario 17. El reverso del psicoanálisis (Buenos Aires: Paidós, 1992).
} 


\section{enn-claves}

Revista de Filosofía, Arte, Literatura, Historia

e-ISSN: 2594-1100, Año XIV, Núm 27, enero-junio 2020, 1-18

de dos tipos de discurso distintos. ${ }^{46}$ Estos dos tipos de discurso no pueden ser ubicados como parte de alguna corriente contemporánea.

[L]a filosofía como una forma de vida es un modelo de filosofía que enfatiza sus aspectos prácticos que cambian la vida. Abarca la división entre la filosofía analítica y la continental, ni alineada ni opuesta a ninguna de las tradiciones. Puede ser una forma útil de pensar la filosofía si la cultura filosófica más amplia tiende a minimizar su lado práctico. ${ }^{47}$

Filosofía como modo de vida y antifilosofía pertenecen a épocas distintas y tienen características diferentes. Para Hadot, la filosofía antigua se comprendía tanto como un discurso teórico como una forma de vida de tal suerte que estas características resultan inconmensurables e inseparables entre sí. Los filósofos antiguos no hacían diferencia entre el discurso y el modo de vida. Por su parte, la antifilosofía es presentada por Alain Badiou en su definición más sucinta como: "cualquier sistema de pensamiento (o pensamiento) que opone la singularidad de su experiencia a la categoría propiamente filosófica de la verdad. De este modo, la antifilosofía vive en algún lugar entre la contradicción entre la filosofía y la actividad pura o el aspecto creativo de la vida". ${ }^{48} \mathrm{La}$ antifilosofía asume la separación irrevocable de la filosofía como teoría pura, y lo que era antiguamente una filosofía que incorporaba el discurso sobre el modo de vida.

Ante la perspectiva de esa filosofía exclusivamente argumentativa, algunos pensadores de la modernidad tardía echan de menos la posibilidad de un discurso que apelara a los intereses del individuo y no de un pensamiento por sí mismo, que sólo tiene una consideración del hombre y la realidad sub specie aeternitatis. Hadot menciona a Schopenhauer y Nietzsche, quienes suponen "auténticas invitaciones a la transformación

\footnotetext{
${ }^{46}$ Gwenaëlle Aubry, "Philosophy as a Way of Life and Anti-philosophy", en Philosophy as a Way of Life, 210-222.

47 "Philosophy as a Way of Life is a model of philosophy that emphasizes its practical, life-changing aspects. It cuts across the division between analytic and continental philosophy, neither aligned with nor opposed to either tradition. It can be a useful way to think about philosophy if the wider philosophical culture tends to downplay its practical side". John Sellars, "What is philosophy as a way of life?", Parrhesia, núm. 28 (2017), 53.

48 “Anti-philosophy is any system of thought (or thinking) which opposes the singularity of its experience to the properly philosophical category of truth. Anti-philosophy thereby lives somewhere in between the contradiction between philosophy and the pure activity or creative aspect of life", en Alain Badiou, Is Lacan An Anti-Philosopher? Seminario en Teoría Crítica Experimental, Schoenberg Hall, UCLA, 28 de mayo de 2010. Transcripción parcial en https://dingpolitik.wordpress.com/2013/06/04/zizek-versus-badiouis-lacan-an-anti-philosopher/; [video] https://www.youtube.com/watch?v=Fv5VMf-RJx4.
} 


\section{enn-claves}

radical de manera de vivir". ${ }^{49}$ Groys menciona que el giro antifilosófico comienza con Kierkegaard y Marx. ${ }^{50}$ Badiou dedica tres seminarios a cada uno de los antifilósofos más importantes: Nietzsche, Wittgenstein, Lacan y Richard Rorty; menciona que en los linderos de la historia de la filosofía moderna: "aparecen figuras que, sin llegar a formar una 'tradición', se parecen entre sí por su desconfianza ante la idea de que la esencia del hombre es ser un conocedor de esencias". 51 Estos autores son llamados por Rorty pensadores edificantes, e incluye a Goethe, Kierkegaard, Santayana, William James, Dewey, el segundo Wittgenstein y al segundo Heidegger.

Es discutible si es lo mismo a lo que se refieren Hadot, Groys, Badiou y Rorty y también cuáles autores exactamente pertenecerían a una misma tradición de antifilósofos o de pensadores edificantes. Lo que sería difícil de discutir, y esto es lo que quisiéramos mostrar, es que en la modernidad tardía surgió una importante inquietud por regresar a las preocupaciones particulares del sujeto concreto en una amplia diversidad de autores. Encontramos esa preocupación común entre aquellos autores que Badiou califica del siguiente modo:

Para el antifilósofo, los dolores y los éxtasis de la vida personal atestiguan que el concepto habita el presente temporal hasta en los tormentos del cuerpo. Y en cuanto a no ser un mediocre, un repetidor, un auxiliar de las gramáticas o un piadoso guardián de las instituciones y los tempos, el antifilósofo se consagra a ello mediante la extrema violencia de lo que profiere sobre sus propios colegas, los filósofos. 52

Difícilmente se podría confundir este estilo con la filosofía tradicional de la modernidad centrada en la argumentación teórica a tal magnitud que Groys y Badiou se deciden por llamar a esta serie de pensadores antifilósofos, en vez de filósofos, y Rorty, filósofos edificantes, en vez de filósofos sistemáticos. ${ }^{53}$ De lo que hablan estos autores es de otra cosa, de un resto.

\footnotetext{
${ }^{49}$ Hadot, Ejercicios espirituales, 245.

${ }^{50}$ Boris Groys, Introducción a la antifilosofía (Buenos Aires: Eterna Cadencia, 2016), 14.

${ }^{51}$ Richard Rorty, La filosofía y el espejo de la naturaleza (Madrid: Cátedra, 1983), 332.

${ }^{52}$ Alain Badiou, La antifilosofia de Wittgenstein (Buenos Aires: Capital Intelectual, 2013), 8.

53 Rorty muestra las diferencias entre estos modos de la siguiente manera: "Los grandes filósofos sistemáticos son constructivos y dan argumentos. Los grandes filósofos edificantes son reactivos y presentan sátiras, parodias, aforismos. Saben que su obra perderá vigencia cuando se pase el periodo contra el que estaban reaccionando. Son intencionadamente periféricos. Los grandes filósofos sistemáticos, como los grandes científicos, construyen para la eternidad. Los grandes filósofos edificantes destruyen en
} 
Esta idea del 'resto' se descubre en toda antifilosofía, que sólo construye redes sutiles de relaciones para acorralar la incompletitud y exponer su resto a la aprehensión por el acto. Y es precisamente allí donde la antifilosofía destituye a la filosofía: mostrándole lo que su pretensión teórica ha perdido, y que no es, en definitiva, nada menos que lo real. ${ }^{54}$

Hay otra cosa, un algo más, un resto al que la filosofía no puede acceder: aquello que no puede ser expresado con símbolos ni con representaciones. Lo que la tradición religiosa conocía como lo inefable y que Lacan identifica bajo su registro de lo real. Nadie puede expresar lo inexpresable, ni siquiera y mucho menos los filósofos. La labor de los antifilósofos es conducir al individuo al contacto con eso real. Mientras que la filosofía se ha cuidado de describir la realidad — como mostró exhaustivamente Rorty en La filosofía y el espejo de la naturaleza-, lo que hacen los antifilósofos o filósofos edificantes es primero reconocer y mostrar que no todo puede ser simbolizado y, segundo, que la experiencia de aquello real es enriquecedora para el individuo. ¿No era ésta la labor de la filosofía en la Antigüedad? Para Platón la reflexión sobre la muerte constituía la esencia misma de la filosofía. Esta reflexión señala el propio límite del sujeto, algo que Lacan refería como un 'resto' inexpresable..

A fin de cuentas, el objeto de la filosofía en la Antigüedad era precisamente el mejoramiento del individuo por medio de las prácticas espirituales. Entonces discurso y filosofía como modo de vida no estaban separados, eran inconmensurables, razón por la cual, Aubry nombró archifilosofía a esta caracterización de Hadot respecto a la filosofía antigua.

Con el advenimiento del cristianismo, la archifilosofía es abolida, pues se separa el discurso teórico de la filosofía como modo de vida cuyas prácticas espirituales son incorporadas a la religión. La característica propia de la filosofía medieval es la supeditación de la filosofía a la teología.

En la modernidad la filosofía se separa de su rol como servidora de la teología y se constituye como un saber propio y autofundado asumiendo plenamente como un

\footnotetext{
beneficio de su propia generación. Los filósofos sistemáticos quieren colocar su materia de estudio en el camino seguro de la ciencia. Los filósofos edificantes quieren dejar un espacio abierto a la sensación de admiración que a veces causan los poetas". Richard Rorty, La filosofía y el espejo de la naturaleza (Madrid: Cátedra, 1983), 334.

${ }^{54}$ Badiou, La antifilosofia de Wittgenstein, 34.
} 


\section{en-claves}

discurso teórico y argumentativo. Por otro lado, el interés del individuo permanece, como herencia de la filosofía medieval, aislado de la filosofía moderna que se desentiende de hacer labores edificantes. Como decía Hegel, el filósofo debe cuidarse de hacer las labores del párroco. ${ }^{55}$

En la filosofía moderna tardía y contemporánea aparecen una serie de pensadores que pueden ser comprendidos bajo los términos de antifilósofos o filósofos edificantes cuyo interés mayor es apelar al mejoramiento del individuo tal y como sucedía en la filosofía antigua. La diferencia entre éstos y los antiguos radica en que el discurso filosófico de la Antigüedad permanecía inseparable de la filosofía, comprendida como un modo de vida, mientras que lo que encontramos en la modernidad tardía son autores que se separan de la que su época considera que es filosofía. No obstante, la preocupación de esta nueva camada de pensadores es la misma que tuvieron los antiguos: la de preguntarse sobre el mejor modo de vivir. Aunque sean conocidos como filósofos, estos pensadores tienen un propósito distinto al de la filosofía tradicional, y por esta razón Badiou y Groys les llamaron más bien antifilósofos.

La inercia social e histórica de la comprensión de la filosofía como un saber académico y eminentemente teórico hacen muy difícil restaurar una unión con la filosofía como modo de vida. La antifilosofía se debe erigir así misma como un discurso autónomo e independiente de la filosofía contemporánea y rescatar el sentido antiguo y más puro de la disciplina tal y como se comprendía en la Antigüedad.

\footnotetext{
55 Georg Wilhelm Friedrich Hegel, Aforismo de Jena, núm. 66, citado en Walter Kaufmann, Hegel (Madrid: Alianza Editorial, 1972), 253.
} 


\section{enn-claves}

\section{Bibliografía}

Aubry, Gwenaëlle. "Philosophy as a Way of Life and Anti-philosophy". Philosophy as a Way of Life: Ancients and Moderns - Essays in Honor of Pierre Hadot, 210-222. Editado por Michael Chase, Stephen R. L. Clark, Michael McGhee. Hoboken: Wiley-Blackwell, 2013.

Badiou, Alain. Is Lacan An Anti-Philosopher? Seminar in Experimental Critical Theory [Seminario sobre Teoría Crítica Experimental]. Schoenberg Hall, UCLA. Mayo 28 de 2010. Transcripción parcial en: https://dingpolitik.wordpress.com/2013/06/04/zizek-versus-badiou-is-lacan-ananti-philosopher/. Video: https://www.youtube.com/watch?v=Fv5VMf-RJx4. Accesado 21 de enero de 2019.

Badiou, Alain. La antifilosofia de Wittgenstein. Buenos Aires: Capital Intelectual, 2013.

Badiou, Alain. Le Seminaire. Lacan. L'antiphilosophie 3. 1994-1995. París: Librairie Arthème Fayard, 2013.

Cavallazzi, Alejandro. “¿Qué es Antifilosofía?”. LÍMITE Revista Interdisciplinaria de $\begin{array}{llllll}\text { Filosofía } & y & \text { Psicología } & 14, & \text { núm. } & 2\end{array}$ (2018), https://www.revistalimite.cl/index.php/limite/article/view/108.

Chase, Michael. "Observations on Pierre Hadot's Conception of Philosophy as a Way of Life". Philosophy as a Way of Life: Ancients and Moderns - Essays in Honor of Pierre Hadot, 262-286. Editado por Michael Chase, Stephen R. L. Clark, Michael McGhee. Hoboken: Wiley-Blackwell, 2013.

Force, Pierre. "The Teeth of Time: Pierre Hadot on Meaning and Misunderstanding in the History of Ideas". History and Theory 50, núm. 1 (febrero, 2011): 20-40.

Groys, Boris. Introducción a la antifilosofía. Buenos Aires: Eterna Cadencia, 2016.

Hadot, Pierre. ¿Qué es la filosofía antigua? México: Fondo de Cultura Económica, 1998.

Hadot, Pierre. Ejercicios espirituales y filosofía antigua. Biblioteca de Ensayo. Madrid: Siruela, 2006.

Hadot, Pierre. Elogio de la filosofía antigua. México: Me Cayó el Veinte, 2009.

Hadot, Pierre. La filosofia como forma de vida, conversaciones con Jeannie Carlier y Arnold I. Davidson. Barcelona: Alpha Decay, 2009. 


\section{enn-claves}

Johnston, Adrian. "This Philosophy Which Is Not One: Jean-Claude Milner, Alain Badiou, and Lacanian Antiphilosophy". Journal of the Jan van Eyck Circle for Lacanian Ideology Critique 3 (2010): 137-158.

Kaufmann, Walter. Hegel. Madrid: Alianza, 1972.

Kierkegaard, Soren. Journals and Papers. Bloomington, Indiana University Press, 1978.

Kierkegaard, Soren. Temor y temblor. Madrid: Alianza, 2001.

Kruger, Matthew. "Aquinas, Hadot, and Spiritual Exercises”. New Blackfriars 98, núm. 1075 (2017): 414-426.

Lacan, Jacques. El reverso del psicoanálisis. Seminario XVII. Buenos Aires: Paidós, 1992.

Lacan, Jacques. "Quizás en Vincennes”. En Otros escritos. Buenos Aires: Paidós, 2012.

Rodolphe, Adam. Lacan y Kierkegaard. Buenos Aires: Nueva Visión, 2007.

Rorty, Richard. La filosofía y el espejo de la naturaleza. Madrid: Cátedra, 1983.

Sellars, John. "What is Philosophy as a Way of Life?". Parrhesia, núm. 28 (2017): 40-56.

Sharpe, Matthew. "Socratic Ironies: Reading Hadot, Reading Kierkegaard". Sophia 55 (2016): 409-435.

Sharpe, Matthew. "Killing the father, Parmenides: On Lacan's anti-philosophy." Continental Philosophy Review 52(2019): 51-74. 